\title{
BIOTECNOLOGÍA EN EL DEPORTE: DEBATE ENTRE LOS BIO- TECNO-FASCINADOS Y LOS BIO-TECNO-FÓBICOS Y SU RELACIÓN CON EL PRINCIPIO BIOÉTICO DE LA AUTONOMÍA ${ }^{1}$
}

\author{
BIOTECHNOLOGY IN SPORT: DISCUSSION BETWEEN BIO-TECHNO- \\ FASCINATED AND BIO-TECHNO-PHOBIC AND ITS RELATION \\ TO THE BIOETHICAL PRINCIPLE OF AUTONOMY
}

Henry David Caro Romero
Luz Amelia Hoyos Cuartas

Resumen

El objetivo de este artículo es realizar un análisis crítico de las dos posturas teóricas predominantes acerca de la incursión de la biotecnología en el contexto deportivo desde el principio bioético de la autonomía. Primero, se estudiarán los argumentos de los bio-tecno-fascinados quienes consideran al imperativo biotecnológico como inaplazable si se puede hacer ...entonces se debe hacer, incluso se aventuran a sobrepasar el principio de precaución, argumentando que en la búsqueda de la mejora deportiva va implícito un cierto grado de riesgo, acercándose a la corriente posthumanista, acorde con el surgimiento de un nuevo deportista mejorado, gracias a los adelantos biotecnológicos aplicados al interior de su cuerpo. En segundo lugar, los bio-tecno-fóbicos se oponen a toda clase de modificación corporal mediada por la biotecnología, cuestionando su uso porque desvirtúa el sentido de lo humano, afecta la competencia leal, y crea ventajas para quienes pueden acceder a dichos avances, frente a quienes no pueden hacerlo. Estas reflexiones, conducen a pensar en la importancia de retomar el principio bioético de la autonomía, ya que es sobre el deportista que recaen los efectos deseados, pero también indeseados, de tales aplicaciones (incluso en ciertos casos, pueden llegar a ser negativos para su salud).

Palabras claves: Tecnología, biotecnología, deporte, principio bioético de la autonomía.

Abstract

The principal aim of this presentation, is to make a critical analysis of both predominant theoretical positions about the biotechnology incursion in the sports context from the autonomy bioethics principle. First able the bio - tecnofascinated, arguments will be studied, who consider deferred the biotechnological imperative if it is possible to do ... then it is necessary to do, even that overpass they risk to exceed the precaution principle, arguing that in the searching improvement the sports goes implicitly a certain degree of risk, approaching in that way to the Posthumanist current, according to the improved sportsman, new emergence, thanks to the biotechnological advances applied in to his body.

Mean time the second group, the bio-tecno-phobic, is opposed to all kinds of corporal biotechnology modification, questioning this use. Because the human sends is spoiled affects the loyal competition, and it creating advantage for those who can accede to the above mentioned advances, opposite to who can accede to the above mentioned advances, opposite to when cannot do it. These reflections, they drive to think about the importance to taking again the Autonomy bioethics mains due to it is over the sportsman relapsed, both the wished and unwanted effects of such applications. (Even in certain cases, they can be health negative effects for him).

Keywords: Technology, biotechnology, sport, the Autonomy bioethics Principle.

Fecha de recepción: 15 de julio de 2014

Fecha de aprobación: 11 de noviembre de 2014

1 Este trabajo hace parte de una investigación doctoral más amplia acerca de los dilemas bioéticos generados a partir del denominado mejoramiento deportivo (Enhancement Sport).

2 Magíster en Bioética, Magíster en Educación y estudiante de tercer año del Doctorado en Educación de la Universidad Pedagógica Nacional. Colombia. Correo electrónico: hcaro42@gmail.com

3 Dra. PhD. Ciencias de la Actividad Física y el Deporte, profesora de planta de la Universidad Pedagógica Nacional. Correo electrónico: luzahoyos@ yahoo.com 
Para citar este artículo:

Caro, H.D., Hoyos, L.A. (2014). Biotecnología en el deporte:

debate entre los bio-tecno-fascinados y los bio-tecno-fóbicos y su relación con el principio bioético de la autonomía. Revista Lúdica Pedagógica, (20), 19-25.

\section{INTRODUCCIÓN}

El deporte en todas sus facetas, pero sobre todo el de alto rendimiento, es un espacio trascendental que ocupa hoy un lugar destacado en la sociedad mundial y cada vez con mayor impacto a nivel latinoamericano. Como nunca antes, la adhesión de personas de todos los niveles y clases sociales; ya sea en forma activa (practicantes) o pasiva (espectadores) va en aumento. De igual manera, la edición de revistas, libros especializados, trabajos académicos, programas de TV. radio, prensa deportiva, intervenciones en eventos de élite mundial y creación de páginas web, contribuyen a la expansión de clubes, academias y asociaciones deportivas, quienes lo promocionan, como un sector clave de la economía de varias regiones y muchos países del mundo.

Sin embargo, el ansia de triunfo, la competencia exacerbada y la necesidad de obtener la victoria también rompe, en muchos casos, los cánones éticos y morales de los participantes, quienes, avalados por sus patrocinadores o desde su propia iniciativa, cada vez están más dispuestos a recurrir a una creciente avalancha de ayudas extras que prometen mejorar el desempeño atlético y cumplir sus sueños de ganar. En medio de ese panorama, el mercado alrededor del deporte empieza a ofrecer un catálogo de productos cada vez más completo, que incluye desde bebidas energéticas y complementos nutricionales, pasando por la utilización de medicinas que fueron elaboradas con fines terapéuticos, pero que podrían aumentar el rendimiento, hasta la disposición de centros de investigación y empresas que trabajan en genómica humana, prometiendo mejoras genéticas desde la línea somática (que corresponde a cambios a nivel celular) o germinal, (que parte de modificaciones a nivel de la estructura genética previa al nacimiento).

\section{BIOTECNOLOGÍA: UN POCO DE HISTORIA}

La biotecnología en el desarrollo de la humanidad es tan antigua como el hombre mismo y se ha ocupado del uso de organismos vivos o de compuestos químicos, con el fin de obtener productos que les permitan a los humanos mejorar su calidad de vida. En sus albores, se limitó a procesos de fermentación de pan, lácteos y bebidas alcohólicas, en lo que podría llamarse una primera generación.

Más adelante, se empiezan a aplicar los conocimientos funcionales de los organismos vivos para promover otras sustancias e innovar técnicas convencionales en el desarrollo industrial, como también se aplican algunas innovaciones en la agricultura y en menor medida en la ganadería. También se reconoce un creciente aumento del trabajo en laboratorio para generar nuevos antibióticos y vitaminas, de allí que esta etapa es denominada por Wilches (2010) como una biotecnología moderna o de segunda generación.

Pero, dado que otras ciencias se han desarrollado al mismo tiempo, en especial la genética, la nanotecnología y la propia tecnología, dichos adelantos hoy le permiten ampliar su campo de acción a una biotecnología de tercera generación, caracterizada por la manipulación de moléculas de ADN, para obtener nuevos organismos y el mejoramiento genético de ciertas plantas y animales, con el fin de alcanzar mayores rendimientos o de protección frente a plagas u otros daños generados por diversos factores, sobrepasando de esta manera las habituales barreras entre especies.

De allí se deriva una cuarta generación, la cual se vale de las investigaciones sobre el genoma humano, que identifica la secuencia en que se encuentran los nucleótidos en el ADN y las proteínas en el organismo humano, abriendo un nuevo espacio para su aplicación en medicina (que recientemente se denomina medicina personalizada), vislumbrando con gran optimismo su carácter preventivo o terapéutico en la salud y el bienestar (Wilches, 2010).

\section{Biotecnología en el deporte}

La incursión de la biotecnología en el deporte es reciente y corresponde a una aplicación no prevista en este campo de carácter interdisciplinar, debida a la creciente necesidad de obtener los primeros lugares en todas las competencias, donde muchos deportistas, y algunos entrenadores, están haciendo uso o estarían dispuestos a someterse como sujetos de experimentación voluntaria, a complejos procesos de modificación genética, dado que hoy la victoria es un suceso que trasciende la satisfacción personal, y es parte de una compleja trama de relaciones económicas, sociales y políticas las cuales se han vuelto comunes en el deporte contemporáneo y donde los reparos éticos son cada vez más problemáticos (Heinemann, 2004). 
Resnik (citado por Drumond, 2013) afirma que uno de los principales dilemas que crean las nuevas opciones de la biotecnología es poder distinguir entre la terapia genética y el mejoramiento genético. Así, mientras en la terapia genética, la finalidad es curativa o regenerativa; en la mejoría genética, el sentido cambia, ya que se busca intencionalmente un incremento de estatura, inteligencia, habilidad musical o, como en el caso del deporte, un aumento de la resistencia, la fuerza o la velocidad, por nombrar solo algunas de sus posibles aplicaciones.

Al respecto, Boladeras (2013) concluye que el impacto de la tecnociencia, el desarrollo tecnocientífico y las repercusiones bioéticas que estos temas generan en diferentes ámbitos, se debe a que van más allá de los límites humanos, ejemplificados en las intervenciones de mejora biogenética que a nivel deportivo empiezan a ser objeto de análisis, por las implicaciones culturales que dichos avances conllevan.

\section{POSICIÓN DE LOS BIO-TECNO-FASCINADOS}

El deporte ha sido, históricamente, un campo de experimentación donde han confluido varias ciencias con el objeto de potencializar los desempeños y alcanzar el éxito. En ese sentido, todo aquello que permita superar las marcas atléticas es rápidamente asimilado y puesto en práctica, sin demasiado énfasis en sus implicaciones éticas. De igual forma, se sabe que disciplinas como la biomecánica, la nutrición, la psicología, y la fisiología se han unido de acuerdo a Dossil (2008) en lo que hoy se denominan como Ciencias de la Actividad Física y el Deporte (CAFD), las cuales desde sus respectivos campos, contribuyen en la preparación de los deportistas, buscando no dejar ningún espacio al azar y tratando de aprovechar al máximo las potencialidades físicas y psicológicas de quienes dedican gran parte de su infancia y juventud en la búsqueda del triunfo.

No obstante, las presiones por ganar, cada vez más elevadas, hacen que la comunidad deportiva recurra también a los avances de otras ciencias, disciplinas o campos de investigación interdisciplinar, como es el caso de la biotecnología, siempre en pro de conseguir los mejores resultados. Posición que está plenamente justificada por todos aquellos autores que defienden desde sus propias disciplinas (filosofía, medicina, bioética, genética y la biotecnología entre otras) la aplicación que, en ciertos casos, implicaría la modificación genética del deportista, a arriesgo de afectar la salud o su futura descendencia, dado que dichas alteraciones son irreversibles.
La argumentación de los denominados bio-tecno-fascinados inicia desde el postulado de que no solo es necesario, sino también imprescindible, dar vía libre a la aplicación de todos aquellos adelantos tecnológicos (algunos externos al deportista) y, más recientemente, los biotecnológicos (que modifican parte de su fisiología), o hasta el punto de pretender hacer cambios estructurales a nivel genético apoyados por la tecnociencia, dejando claro que aunque desde el inicio de la humanidad, el ser humano siempre ha tratado de aplicar a otros, o a sí mismo, diversas técnicas en procura de superarse y en esta época la idea sigue vigente, y lo único que cambia son los medios para lograr dicho objetivo.

\section{La tecnología como parte inherente al deporte}

\section{Antecedentes}

Desde la antigüedad, los deportistas han utilizado algún tipo de ayuda tecnológica con el fin de aumentar su rendimiento, así, por ejemplo, dice la tradición que los luchadores grecorromanos guardaban en pequeños recipientes la mezcla de sudor y arena que quedaba en sus cuerpos después de las contiendas, poción que luego era vendida a los menos diestros, con el ánimo de que su aplicación aumentara su fuerza y combatividad. También se sabe que numerosas combinaciones nutricionales elaboradas desde los cánones de la medicina hipocrática eran usuales en la preparación de los deportistas.

Siguiendo esta misma línea, las civilizaciones posteriores han combinado diversos métodos, unos más naturales, otros más artificiales, que se pueden denominar en palabras de Posada (2000), parte de la artificialeza (combinación de lo artificial con lo natural) en que se encuentra el hombre moderno con el propósito de mejorar su desempeño. Ahora bien, con respecto a los grados de intervención de la tecnología, estos también han ido variando en el tiempo; así la indumentaria, los implementos y artefactos son cada vez más sofisticados, como también el uso de bebidas y dietas especiales para disponer del máximo de energía a ser utilizada en los entrenamientos y las competencias.

\section{Actualidad}

En la contemporaneidad, la influencia de la tecnología es absoluta y necesaria para mantener la vigencia competitiva, así, algunos atletas por su condición económica pueden acceder a un sinnúmero de aparatos. A continuación, se describen algunos de estos, empezando por 
el criosauna, una adaptación de una sauna convencional con la particularidad de que permite al deportista someterse a bajas temperaturas para acelerar los procesos de recuperación. También es cada vez más frecuente el uso de la cámara hiperbárica, para aumentar el aporte de oxígeno a los tejidos, mejorando la capacidad de transporte de la hemoglobina, la cual resulta determinante en el tratamiento de las lesiones y permite un mayor descanso.

En ese mismo orden, hace poco se lanzó al mercado el llamado Alter G. o Anti-Gravity, que consiste en una cinta rodante modificada, la cual admite experimentar sensaciones antigravedad, haciendo posible que atletas lesionados puedan correr para no perder la forma física, ya que su tecnología está configurada, para experimentar solo el $20 \%$ de su peso corporal. Otro aparato similar es el Batak Pro Machine, donde el deportista entrena la velocidad de reacción para mejorar sus reflejos, a ellos se unen el EuroGoal: una máquina para lanzar balones a los arqueros de fútbol (similar a la que se utiliza en tenis o béisbol). Como se puede intuir, la lista seguirá aumentando día a día de forma exponencial.

De igual manera, los controles tecnológicos que se ejercen en el deporte hoy son cada vez más precisos, el denominado ojo de halcón utilizado en tenis, hoy pasa al fútbol. Asimismo, los comunicadores inalámbricos que permiten a los árbitros decidir más acertadamente son una muestra de la gran variedad de artefactos que poco a poco han ido ganando un espacio en el deporte, dado que los resultados deportivos están ligados a la parte económica y una equivocación resulta, literalmente, demasiado costosa para el perdedor, pero sobre todo, para los patrocinadores, la TV., y los publicistas entre otros.

Ahora bien, cuando las tecnologías se plantean con un carácter invasivo (como es el caso de las modificaciones biotecnológicas), surgen otros interrogantes y numerosos dilemas bioéticos difíciles de resolver, dado lo inédito de tales alteraciones $\mathrm{y}$, aunque algunas solo sean planteadas a nivel hipotético, causan una mezcla de admiración, estupor y cierto temor en la comunidad deportiva. Allí son otros los presupuestos que entran en juego, se trata de cambiar estructuralmente al deportista para que aumente su rendimiento, lo cual no se limita al espacio de la vida deportiva que es corto en el tiempo, sino que será por toda la vida, e incluso con posibles beneficios o daños a terceros (en el caso de su descendencia).
Al respecto, Scott (2004) afirma que aunque son numerosas las objeciones éticas acerca del mejoramiento de las capacidades humanas por medios artificiales (incluidas las deportivas), estas han sido insuficientes para disuadir su empleo, en especial por los intereses extradeportivos (económicos) que hoy determinan de forma imperativa lo que se debe hacer en el deporte de alto rendimiento. Por esta razón, se aventura a proponer que una posible salida sea dar un espacio a nuevas perspectivas del pensamiento ético convencional, que por siglos ha respetado el orden natural dado por la herencia genética, pero que no contaba con las posibilidades que la ciencia y las recientes biotecnologías vislumbran como aplicables en el campo deportivo.

Siguiendo el mismo esquema, Tamburrini y Tännsjö (2012) afirman que la manipulación genética, puede ser un camino inédito para superar (o al menos tratar de igualar) la natural desventaja entre los deportistas, lo mismo afirman Savulescu, Foddy y Clayton (2004), asumiendo un punto de vista diferente al tradicional, que se ocupa de juzgar negativamente esta nueva tendencia que cada vez se percibe como una realidad en el corto plazo para el deporte de alto rendimiento.

Es de anotar que la publicidad y los medios de comunicación, con fines de aumentar la audiencia, se han encargado de magnificar experimentos biogenéticos, muchos de ellos, en las primeras fases y solo probados en laboratorio con animales. Esto ha tenido efectos insospechados; en palabras de Mejía (2010), en la actualidad algunos padres obnubilados por la propaganda tratan de persuadir al cuerpo médico para que a sus hijos les sea aplicada la hormona de crecimiento, con el objetivo de mejorar las posibilidades de ser elegidos, en el caso de que intentaran postularse como deportistas.

\section{POSICIÓN DE LOS BIO-TECNO-FÓBICOS}

Dada la complejidad de las diferentes culturas, no resulta fácil convencer a todo el mundo de las bondades de la biotecnología aplicada al deporte, y más cuando muchas de esas promesas han sido creadas a partir de supuestos posibles, pero no comprobables por ahora (quizás en un tiempo no muy lejano, esto efectivamente se pueda realizar). Las objeciones vienen de muchas fuentes, este escrito está centrado en el punto de vista de algunos filósofos y bioeticistas que han investigado en este campo y quienes se valen de un repertorio amplio de argumentos para cuestionar su uso por considerarla contraria a los mandatos de sus convicciones personales, o por conflicto de intereses. 
Para iniciar, Trainor (2012) afirma que cuando este tipo de investigación se lleva a cabo para sujetos determinados (en este caso, deportistas) su relevancia es cuestionada por el poco valor que representa para la sociedad en su conjunto, incluso podría ser una desviación de recursos que bien estarían mejor empleados con fines más altruistas, y en cambio sí se pondría en riesgo a las personas que participan en dichos estudios. Otro aspecto que es cuestionado sería que si estas investigaciones son financiadas con recursos del Estado, pero se aplican de manera privada, solo para quienes puedan pagarlas, de cierta forma es una apropiación indebida de los bienes públicos.

Por su parte, Beauchamp y Walter (citados por Arellano y Hall, 2012) afirman que James D. Watson, premio Nobel y codescubridor de la estructura del ADN, presenta varias objeciones para la aplicación de la biotecnología que deberían ser tenidas en cuenta: 1) La precisión de las pruebas genéticas, y 2) El consentimiento informado para las investigaciones y tratamientos de la mejora humana.

Lo anterior es aplicable con ciertas diferencias al deporte de alto rendimiento, por ejemplo, en cuanto a la precisión de las modificaciones genéticas, una cosa es plantear el tema desde fuera y otro es lograr en el laboratorio el control efectivo en la implantación de un gen sin modificar o alterar aquellos que lo circundan, y después coordinar diferentes factores para que se produzca el resultado esperado, el cual sigue estando mediado por un sinfín de eventos unos previsibles y otros incontrolables. Por esto, se hace imprescindible también el reflexionar sobre los costos y beneficios que estas decisiones implican.

Con respecto al consentimiento informado, es importante resaltar que se ofrezcan las precauciones adecuadas para los sujetos que se sometan a este tipo de investigaciones. Por ejemplo, Trainor (2012) afirma que históricamente los participantes no han tenido garantías suficientes de protección para su salud ante el riesgo que esto implica, resultando en muchas ocasiones afectados severamente, y todo en nombre de la investigación científica. Esto se debe, en gran parte, a las marcadas diferencias de conocimiento entre los participantes y el experimentador, quien podría omitir información clave para que ellos cooperen; además, cuando se trabaja con grupos sociales vulnerables el riesgo aumenta de forma elevada, por las posibles dependencias en que estas personas tienden a incurrir.
Por estas razones, se requiere de un máximo control para evitar abusos de parte de quienes dirigen estas investigaciones, tratando de que sean los propios sujetos y los grupos a los que ellos pertenecen, quienes salgan beneficiados y no solo quienes las plantean. Enlazado con lo anterior, si bien es cierto que la aplicación de la biotecnología en el contexto del deporte promete grandes adelantos y facilitaría el logro de mejores desempeños, no se puede dejar de lado su sostenibilidad, ya que al ser tan reciente adolece de una base amplia de investigaciones y está en riesgo de producir efectos inesperados, alteraciones no previstas y consecuencias irreversibles, afectando en el largo plazo la salud del deportista.

Ahora bien, si se mira de manera realista, dichos planteamientos vendrían a dar un segundo aire a la eugenesia, que de manera indirecta se estaría reviviendo con otros fines. Siguiendo de nuevo a Arellano y Hall (2012), hay una reserva de la sociedad actual en gran parte justificada por el historial negativo que esta tendencia ha tenido de aceptar cualquier intento de mejora humana, partiendo de los supuestos beneficios para la sociedad que en realidad se limitarían a un solo grupo.

\section{FUNDAMENTACIÓN TEÓRICA DE LA BIOÉTICA}

En esta segunda parte, se hará una breve referencia a la definición de la bioética como disciplina académica, abordando luego una explicación sobre los cuatro principios que sustentan la propuesta de la bioética principialista anglosajona, que es la que mayor impacto ha tenido en el mundo (no maleficencia, beneficencia, justicia y autonomía), centrando la mirada en el principio del respeto a la autonomía, en relación con la aplicación de la biotecnología en el deporte de alto rendimiento.

Se afirma, para iniciar, que la denominación de principio en el caso de la bioética, sirve como una guía en la toma de decisiones complejas y situadas en un contexto específico, pero que no funciona como lo hace en el ámbito científico, donde su alcance se supone universal y con un alto nivel predictivo.

Bioética: una definición

El filósofo Gilbert Hottois (2007) hace una aproximación de una posible definición contemporánea de la Bioética en los siguientes términos:

La Bioética cubre un conjunto de investigaciones, de discursos y de prácticas generalmente pluridisciplinarias y pluralistas, que tienen como objeto aclarar y si es posible resolver preguntas de tipo 
ético suscitadas por la investigación y el desarrollo biomédico y biotecnológico [énfasis del autor] en el seno de sociedades caracterizadas en diversos grados, por ser individualistas, multiculturales y evolutivas. (p. 17)

Resulta evidente que la propuesta de la bioética es servir de mediadora entre diferentes posturas teóricas acerca de los efectos reales o potenciales que la investigación biomédica y biotecnológica está produciendo en la actualidad, aclarando que no siempre se puede llegar a consensos, en especial por la multiplicidad de intereses de quienes lideran estos campos científicos, en los cuales las variantes extradeportivas tiene una marcada influencia. De allí que es necesario sopesar los factores que están en juego en el campo deportivo, donde, de igual manera, la tendencia es a colocar lo económico por encima de cualquier otra consideración.

\section{Principios bioéticos}

Los principios bioéticos se consideran generalizaciones normativas que deben servir para guiar la conducta en diferentes contextos donde el sujeto tenga que tomar decisiones que impliquen relaciones con aspectos referentes a dilemas éticos (como en este caso, el contexto deportivo), pero cuya aplicación concreta a casos particulares debe atender las circunstancias específicas. El hecho de ser declarados de prima facie (a primera vista) significa que no existe entre ellos un orden jerárquico omnipresente y que su aplicación deberá ser flexible y ajustada a casos únicos, permitiendo el compromiso, y la negociación consensuada, en la búsqueda de decisiones originales sin la obligación de recurrir siempre a una aplicación diferenciada y preestablecida de antemano. A continuación se hace una breve descripción de cada principio y su interpretación en el contexto deportivo.

\section{a) Principio de no maleficencia}

Se refiere a la obligación de no hacer daño y se sintetiza con la frase primum non nocere. En este momento en el contexto deportivo, y dadas las nuevas alternativas que brinda la biotecnología aplicada al deporte, la exigencia de cuidado aumenta en el sentido de que muchas de sus potenciales aplicaciones, van más allá de la terapia y podrían afectar la calidad de vida de los deportistas.

\section{b) Principio de beneficencia}

Caracterizado por la búsqueda del bien y la protección de los derechos de quienes sean sus usuarios, al igual que la obligación de socorrer y tomar las mejores decisiones que requieran análisis de costo-beneficio, evitando tratamientos y medidas ineficaces o fútiles. Resumiendo, la beneficencia en el deporte se define como la acción moral de actuar en beneficio de los deportistas, aunque no sea obligatorio hacerlo.

\section{c) Principio de justicia}

Este principio encierra enfoques tan diferentes como filosofías o planteamientos ideológicos se adopten. Y, aunque no hace referencia a un concepto penal, civil o rectificador, está encaminado y se supone corregirá o contribuirá a colmar la falta de oportunidades de quienes estén en inferioridad, sean más débiles o se encuentren por sus condiciones socioeconómicas o de cualquier otra índole en una situación de clara desventaja, buscando, en todo caso, dar a cada quien lo que le corresponda.

d) Principio de respeto a la autonomía

Su aplicación se abre paso en el actual entorno deportivo, con muchas implicaciones referentes a la capacidad de tomar decisiones de los deportistas, buscando armonizar las contradicciones y los conflictos inherentes al deporte y que en ocasiones resultan irreconciliables. Por estas razones y de manera prudente, una teoría acerca de la autonomía de los atletas que exija un ideal fuera del alcance del resto de la población no debería de entrada considerarse como válida.

En consecuencia, se propone para la aplicación del principio del respeto a la autonomía las siguientes condiciones: a) las decisiones autónomas del deportista son decisiones intencionadas y debe existir claridad de sus alcances presentes y algunos indicadores a futuro; b) todas las decisiones deben ser tomadas con conocimiento de la actuación deportiva que se plantee, de su significado y de las múltiples consecuencias que de ella puedan derivarse; c) las decisiones deberán ser tomadas a ser posible en ausencia de coacciones externas al deportista; y d) es necesario desarrollar procesos educativos y formativos de autorregulación en los deportistas, de tal forma que sus decisiones busquen no solo su propio beneficio particular, sino también contribuyan al bien común.

En este orden de ideas, es necesario retomar el tema de consentimiento informado en el deporte, el cual encaja con el principio de autonomía, ya que en los procesos de toma de decisiones que impliquen la ingesta, modificación corporal o genética de los deportistas, los protocolos deberán ser extremadamente claros y precisos en la descripción de los elementos que deben formar 
parte de dicho consentimiento, es decir, ser especificadas las condiciones iniciales, el componente informativo acerca de los procedimientos que se llevarán a cabo, además de los posibles riesgos y los elementos de consentimiento o de no consentimiento por parte del deportista, y, finalmente, la posibilidad de abandonar el estudio en cualquier momento, en especial, cuando se perciba una afectación en la salud o la calidad de vida.

\section{CONCLUSIONES Y REFLEXIONES FINALES}

- La instalación de la tecnología y todas sus derivaciones, incluida la biotecnología en el deporte de alto rendimiento, es un hecho innegable que, en adelante, deberá ser objeto de múltiples investigaciones y reflexiones por parte de la comunidad deportiva.

- Por el momento los bio-tecno-fascinados están liderando el debate de la inclusión de la biotecnología en el deporte, y la perspectiva es que lo seguirán haciendo, dada la irrefrenable capacidad del hombre de producir y aplicar artefactos, incluso en sí mismo.

- Es necesario informar y preparar a los deportistas en temáticas de la bioética, y en especial, sobre el principio del respeto a la autonomía y el consentimiento informado, para evitar los abusos que, en un pasado reciente, han tenido lugar en nombre de la ciencia.

\section{REFERENCIAS}

Arellano, J., y Hall, T. (2012). Bioética de la biotecnología. Querétaro, México: Fontamara.

Beauchamp, T., y Childress, J. (2001). Principios de ética biomédica. Barcelona: Masson.
Boladeras, M. (2013). El impacto de la tecnociencia en el mundo humano. Barcelona: Tecnos.

Dossil, J. (2008). Psicología de la actividad física y el deporte. Madrid: McGraw Hill.

Drummond, J. (2013). Ética, bioética y los desafíos del siglo XxI. Derecho PUCP, (69), 65-79.

Heinemann, K. (2004). ¿Es sostenible el deporte actual? Un análisis desde la perspectiva ética Dossier: El deporte: dialogo universal. Apunts 78(4), 10-18.

Hottois, G. (2007). ¿Qué es la Bioética? [Traducido por Chantal Aristizabal]. Bogotá: Universidad El Bosque.

Mejía, R. (2010). En el jardín de Mendel: Bioética, genética humana y sociedad. Medellín: Universidad de Antioquia.

Posada, R. (2000). Bioética: Una visión ética del manejo del propio ambiente. Medellín: Universidad Pontificia Bolivariana.

Savulescu, J., Foddy, B., y Clayton, M. (2004). Why we should allow performance enhancing drugs in sport. British Journal of Sport and Medicine, 38, 666-670.

Scott, R. (2004). The inevitability of genetic enhancement technologies. Bioethics, 18(1), 1-26.

Tamburrini, C., y Tännsjö, T. (2012). The Ethics of Sports Medicine. London: Routledge.

Trainor, B. (2012). The Ethics of Biomedical Enhancement Research (Tesis de maestría). Carleton University. Recuperado de: https://curve.carleton.ca/ system/files/theses/31487.pdf

Wilches, F. (2010). La biotecnología en un mundo globalizado. Revista Colombiana de Bioética, 5 (2), 164-169. 